\title{
QoE Estimation of a Location-Based Mobile Game using on-body sensors and QoS-related data
}

\author{
Adrián Juan Verdejo ${ }^{1}$, Katrien De Moor ${ }^{2}$, Istvan Ketyko ${ }^{1}$, Karen Torben Nielsen ${ }^{3}$, Jeroen Vanattenhoven ${ }^{4}$, Toon De \\ Pessemier ${ }^{1}$, Wout Joseph ${ }^{1}$, Luc Martens ${ }^{1}$, Lieven de Marez ${ }^{2}$ \\ ${ }^{1}$ Department of Information Technology, Ghent University / IBBT, G. Crommenlaan 8, 9050 Ghent, Belgium \\ ${ }^{2}$ Department of Communication Sciences, Ghent University / IBBT, Korte Meer 7, 9000 Ghent, Belgium \\ ${ }^{3}$ IBBT-SMIT, Vrije Universiteit Brussel, Pleinlaan 2, 1050 Brussels, Belgium \\ ${ }^{4}$ Centre for User Experience Research, IBBT / Katholieke Universiteit Leuven, Parkstraat 45, 3000 Leuven, Belgium
}

\begin{abstract}
The scope of this paper is the interdisciplinary measurement and modeling methodology of Quality of Experience (QoE) when playing a mobile location-based massively multiplayer online role-playing game (MMORPG) that places the virtual world on top of the real world using the user's location. The paper introduces the implementation of a re-usable mobile QoE measurement framework on the Android platform and illustrates how it was applied in a concrete case-study. In this respect, a multidimensional QoE prediction model consisting of both objective and subjective parameters is presented. In this model, test users' evaluations regarding feelings of amusement, absorption or engagement experienced while playing a popular location based mobile game (Parallel Kingdom) are taken into account and related to a set of objective QoS-related parameters, contextual data, and physiological data obtained from an on-body sensor. The latter evidences the intensity of physical activity of the test users during the gaming session, taking into account Metabolic Equivalent of Task (MET) measurements. Results of this study indicate that the test users' QoE was influenced by their physical effort, the data connection type used, and the playing context (e.g. interaction with other players). Despite of the fact of using a re-usable framework for QoE estimation, the analysis is limited to this particular popular (more than 100000 users) mobile game.
\end{abstract}

QoE; QoS; Google Android platform; on-body sensor; 3G; location-based (LB) mobile game; massively multiplayer online role-playing game (MMORPG)

\section{INTRODUCTION}

Over the last years, the interest in the Quality of Experience (QoE) concept has grown steadily, both in the industry and in academia. Moreover, research focusing on the quality of the experiences that ICT users have with (new) products or services seems to have evolved to a study field on its own. Topics that are discussed in this relatively new research community include the definition and elements of QoE, the evaluation of both standardized and new measures and measurement approaches, etc. In the scope of this paper, we are particularly interested in those measurement approaches that try to bridge the gap between objective, technical aspects, physiological data and subjective, user-related dimensions influencing QoE. The former are usually incorporated in definitions of QoE from a telecommunications perspective in which QoE is seen as an extension of the traditional Quality of Service (QoS) [1], thus ignoring the subjective character of a person's experiences. In the literature, the lack of attention to this subjective character is criticized. In [2] it is proposed to use the term QoE as an umbrella term, focusing on the broad range of aspects (e.g., usage context, expectations, previous experiences, and network conditions) that might have an influence on QoE. This conceptual idea corresponds with [3], situating QoE-research in a wider "communications ecosystem".

In this paper, we present results from an empirical study that combines objective and subjective aspects of QoE related to the use of Parallel Kingdom, i.e., a mobile location based real-time massively multiplayer online role-playing game (MMORPG) that places the virtual world on top of the real world using the user's location and that can be played on the Android mobile framework. Our aim is to present a methodology to estimate the overall QoE and other subjective parameters related to absorption into a game, and the experience of fun and engagement (explicitly evaluated by test users) of location based mobile MMORPGs, taking into account QoS-related parameters, players' environmental context, and physiological data (e.g. the amount of moderate exercise performed by the user) gathered by on-body sensor and on the other the and overall QoE (explicit evaluation by the test users). The presented analysis is limited to this particular popular mobile game.

\section{RELATED WORK}

Our everyday life is becoming increasingly saturated by mobile devices with higher resolution displays, increased power and faster communications. Commercial location-based services that run on these devices are becoming more and more established. At the same time, research into new locationbased services continues to move forward, in areas such as recommendation [4], cultural tourism [5], and games [6]. Location-aware technology and Internet connectivity embedded in mobile phones allow users to navigate physical spaces and to be connected to other users, bringing locationbased mobile games to physical hybrid spaces by using urban spaces as the game scenario $[7,8]$ and changing the interaction with the city, as well as with other users [9]. There is some literature concerning QoE of online games, focusing mostly on performance issues (related to delay and jitters) of first-personshooters (FPS) [10]. Nevertheless, we did not find any paper in the literature focusing on QoE of location-based mobile MMORP games, which involve user interaction at a slightly slower pace than the aforementioned FPS games. Therefore, the former are less likely to be delay-sensitive than the latter. 
Researchers in Human Factors have used physiological measures as indicators of mental effort and stress [11]. Psychologists use physiological measures as unique identifiers of human emotions such as anger, grief, and sadness [12]. Some experiments aim at correlations between a player's physiological state, events occurring during the entertainment experience, and the subjectively reported experience [13]. However, physiological data have not been employed to identify nor QoE neither user experience states such as boredom or absorption while playing a mobile game.

As far as we know, this is the first paper to present a model for QoE and other subjective parameters (e.g. absorption into the game) of location based MMORPGs focusing in physiological data and QoS-related parameters as well as in the interaction between users playing together in real-time.

\section{MEASUREMENT SetUP}

\section{A. Concept}

Our purpose is the measurement of QoE when playing Parallel Kingdom (PK) [14], a mobile location based massively multiplayer real-time game available for Android devices (downloadable via Google Market), iPhone, and iPod Touch. Three QoE dimensions such as: QoS, context, and user experience [2] are taken into account besides physiological data logged by a SenseWear BMS [15], a body monitoring armband. After being briefed about the purpose and rules of the mobile game, users were asked to play PK outdoors for an hour while the QoE Agent installed into the device was monitoring and logging QoS, physical and contextual parameters. In order to become aware of User Experience, the QoE Agent firstly showed a survey after half an hour of gaming and then the same survey after another half an hour.

\section{B. Application}

1) Hardware and software environment:

Smartphones used in this experiment were both the HTC Android Developer Phone 1 (ADP1) and the Google IO Device with a 1.6 version of Google Android Operating System (donut) installed on them. These devices are capable of running native applications, allowing the integration of common Linux tools into the QoE measurement framework. Specifically, the tcpdump [16] packet analyzer is used to collect network-level QoS data on the device.

\section{2) QoE Measurement Architecture:}

Figure 1 explains the deployment of the QoE measurement architecture into four nodes (namely Android Mobile Device, QoS Data Back-end, Parallel Kingdom Server and Google App Engine) according to the UML standard 2.2. The functional software entities in the nodes are called components. Three components (namely Parallel Kingdom, QoS \& Context Monitor and Experience Monitor) are deployed into the Android Mobile Device node and three components (namely QoS Data Analyzer, Survey Back-end and PK Virtual World) are deployed into three different server nodes (Figure 1). The communication between the components deployed on the different nodes is performed through two interfaces (namely IUpload, IAggregate and IPK indicated by circles in Figure 1).

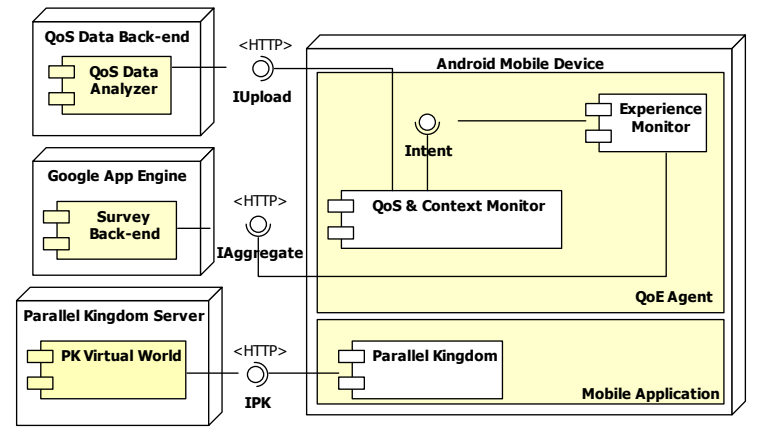

Figure 1. Deployment diagram of the QoE measurement architecture.

We will now explain the components deployed on the Android Mobile Device node:

- Parallel Kingdom Mobile component is a mobile location based real-time MMORPG. $P K$ mobile connects to $P K$ server in order to authenticate the player and download all the data about users' profiles and the virtual world (e.g. monsters' location and skills, and resources positions). This component is fully independent of those components belonging to the QoE Agent.

- The QoS \& Context Monitor component logs network QoS, physical and contextual parameters to files, which are automatically transferred to the QoS Data Analyzer component via the IUpload interface.

- The Experience Monitor component displays surveys and automatically uploads filled-in surveys through the IAggregate interface to the Survey Back-end component deployed on the Google App Engine node.

3) Parallel Kingdom

Parallel Kingdom is the location-based real-time mobile MMORPG (Massively Multiplayer Online Role-Playing Game) the users where asked to play. The objectives of PK are similar to those in every MMORPG game: establishing cities, waging wars, claiming territories, hunting creatures and exploring dungeons. In order to do that the player is able to master 9 different skills, collect and craft items, trade and make alliances with other real users, wield weapons, harness minions and sail the seas.

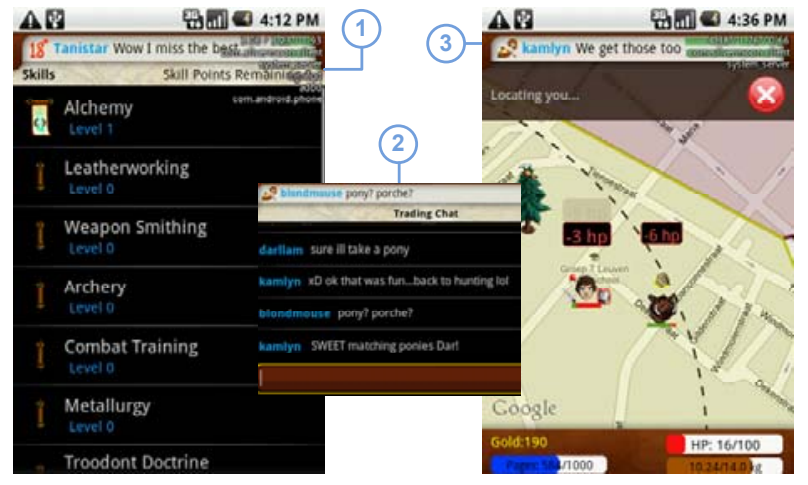

Figure 2. Screenshots of Parallel Kingdom mobile game. 
In Figure 2, a collection of screenshots of Parallel Kingdom are shown. When the user first begins playing, both his/her circle of mobility (dotted line in screenshot number 3 in Fig. 2) and character are centered at his/her GPS/Wi-Fi location. The players can move their character within their circle of mobility by tapping the touch screen. However, in order to access new areas they should move in real life, permitting the application to relocate them and letting them to explore new territories (screenshot number 3 in Figure 2). That made it interesting to investigate whether the physical movement of the user in the real world would influence user's QoE. As in any other MMORPG, interaction between players and characters evolution are very important. PK players are able to interact by chatting (in dedicated specific chat rooms) in order to pursue common goals, sell and buy items, etc. (screenshot number 2 in Figure 2). A user can track its character's mastered skills and level of knowledge of these skills (screenshot number 1 in Figure 2), inventory, alliances or buildings in his possession.

\section{Observed Parameters}

In this experiment there are 2 sources of data: the QoE Agent and the on-body sensors. All this data gathered is studied in order to provide an estimation of subjective parameters using measured objective data. The QoE Agent provides data regarding context (i.e., location and user movement) besides objective and subjective (user-related) parameters. Contextual and objective parameters (i.e., measured QoS parameters) are logged by the QoS \& Context Monitor component; on the other hand, subjective parameters are logged by the Experience Monitor component. Further, the on-body sensors enable automated monitoring of energy expenditure, activity, and sleep efficiency. These sensors provide physiological data related to the user.

\section{1) Objective parameters from the QoE Agent}

We will explain in this section a list of objective parameters used for the statistical analysis. These parameters were calculated for every user's mobile gaming session based on the data logged by QoE Agent. Every parameter was sampled once per second. The time percentage of the gaming session the user was connected to a specific data network type is logged into 4 different variables: $W P, G P, E P$ and $3 P$ (Wi-Fi, GPRS, EDGE and $3 \mathrm{G} \& \mathrm{~B} 3 \mathrm{G}$ data network type, respectively). $W R, G R, E R$, $3 R$ represent the arithmetic average of the observed received signal strength indication (RSSI) values sampled in each second for the four data network types already enumerated. RSSI is here measured in $\mathrm{dBm}$ (from -113 to $-51 \mathrm{dBm}$ ), which is an abbreviation for the power ratio in decibels of the measured power referenced to one milliwatt. $H O$ (number of handovers) describe all kind of radio cell reselection whereas ISHO (Inter-System Handovers) stands for the number of handovers between different data connection-type cell reselections (e.g., between UMTS and GPRS).

Mobility speed is measured in $[\mathrm{m} / \mathrm{s}]$ based on GPS information. Four mobility (M) categories are determined: indoor (no GPS signal detected), no $(0 \mathrm{~m} / \mathrm{s}$ speed), slow (below $6 \mathrm{~m} / \mathrm{s}$ ) and faster mobility (above $6 \mathrm{~m} / \mathrm{s}$ ). The percentage shares of these mobility categories were $3.7 \%, 57.7 \%, 38.6 \%$, and $0 \%$ for indoor, no, slow, and faster mobility, respectively. The QoE agent was continuously monitoring GPS coordinates as well (longitude, latitude, and altitude).

\section{2) Objective parameters from the on-body sensors}

In order to monitor user's physiological responses we attached to each user a SenseWear Body Monitoring Solution armband during the whole gaming experience. Prior to the experiment, these devices were configured according to the user's age, gender, weight $(\mathrm{kg})$, and height $(\mathrm{cm})$. As a result, among other parameters the Metabolic Equivalent of Task in Kcal was provided per minute. Users' physical activity while playing $P K$ was inferred from the Metabolic Equivalent of Task. Thresholds were set to distinguish between light ( 0 to 3 METs), moderate (3 to 6 METs), vigorous (6 to 9 METs), and very vigorous exercise ( 9 METs and higher) [17]. This information was utilized to determine which percentage of the time the player was either sedentary (light exercise $(L E P)$ ) or undergoing physical exercise (either with moderate, vigorous, or very vigorous intensity). $M E P$ is the amount of moderate exercise performed by the user while playing PK. In this experiment, $M E P$ equals the subtraction of $L E P$ (light exercise) from $100 \%$.

\section{3) Subjective parameters}

Subjective parameters were gathered via two identical mobile questionnaires automatically shown to the user both after 30 minutes and one hour of gaming. These subjective parameters were measured on a 5-point scale, meaning 1 the worst score and 5 the best. From these surveys, we extracted some of the users' feelings while playing the game regarding how absorbed into the game they felt (Absorption, A), how bored they were (Boredom, $B$ ), and how enriching they found the experience $(Q o E)$. They were also queried on whether the game required them to make a lot of effort or not and to what extent $P K$ fulfilled their expectations. In order to investigate the influence of the context on the QoE we collected information about how many people (excluding the players themselves) were close to the user in the real world and about how many other players were around the test user in the virtual world $(P)$.

\section{Experiment and procedure}

Using the convenience sampling method, we recruited 28 test users for the experiment. This number of users is relevant to draw conclusions as will be shown in section E. Firstly, the test users were briefed (in small groups of four to five people) about how to play PK and the purpose of the game itself Secondly, they watched the mobile game trailer. Next, they filled in a short general questionnaire including questions about their socio-demographical context, their first expectations about the mobile game and their expertise using Smartphones, playing MMORPG and games in general. Then, every user received an android device. From then on, they played Parallel Kingdom for 1 hour in groups of either four or five people. A body monitoring armband was attached to every user while they were playing the location-based mobile game. The objective parameters coming from both the QoE Agent and the on-body sensors were monitored during the whole gaming session. A survey was filled in after half an hour of gaming and the same survey after another half an hour. 
Finally, those players who played at the same time were interviewed all together. They were required to talk about whether they like the game, their expectations, and to what extent those were met or not.

\section{E. Analysis and results}

The total number of observations is 56 , from the 28 test users ( 2 observations per user). A sample size of 56 is considered sufficient for the central limit theorem to hold and ascertain normality of the residuals and estimated regression coefficients following from linear regression [18]. By this, the distributional assumptions of the t-tests for the significance of regression coefficients are satisfied, which makes these tests meaningful. The dataset of the experiment was analyzed using the statistical software package IBM SPSS Statistics 18.

\section{1) Connection Type}

These experiments were performed within the Proximus UMTS/HSPA network in Belgium. Test users could freely choose between Wi-Fi and cellular data connection. However, none of the users selected a Wi-Fi access network. Mainly, 3G \& B3G data connection was used with a $93.39 \%$ share. In a minority of the cases, no $3 \mathrm{G}$ or $\mathrm{B} 3 \mathrm{G}$ connection was available, which forced the device to switch to the slower-bandwidth GPRS or EDGE solution. This data connection type has a direct influence on the bandwidth of the communication link between the game server and the mobile device.

\section{2) Correlations}

Evaluating QoE of location based mobile MMORPGs is challenging since QoE might be farther affected by enjoyment, expectations, context and interaction rather than productivity, QoS or performance [13]. This is the case most of all for, for games with low CPU, network, and graphic requirements (e.g., Parallel Kingdom). On the other hand, communication and interaction between players could have a large impact in overall QoE of PK, since players can collaborate in order to hunt monsters, form alliances or conquer territories as happens in any other MMORPG. In addition, new territories can be explored by the user's physical movement in the real world. Thus, we wanted to investigate the influence of physical movement and exercise on the overall QoE and the user's emotions.

Table I lists significant correlations between some objective and subjective parameters, showing the significance (p) of the correlation ( $\mathrm{r}$ stands for its correlation coefficient) between two parameters. All listed parameters have a highly significant (either positive or negative) correlation with the amount (in percentage) of moderate exercise performed by the user while playing PK ( $M E P=100-L E P$ (in this experiment)). Regarding the QoE and Boredom $(B)$, both are influenced by both the number of players the user can interact with $(P)$ and the GPRS connection share $(G P)$. On the other hand, no significant correlation was found between the absorption felt by the user while playing a location-based MMORPG (Absorption, $A$ ) and neither $G P$ nor $P$, hence neither the significance of the correlation nor the correlation coefficient appear in Table I. As for the rest of the objective parameters from the QoE Agent (section III.C.1) and those logged by the on-body sensors (section III.C.2), no significant correlation was found with the subjective parameters. Therefore, they are not presented in the following models.

TABLE I. CORRELATIONS BETWEEN OBJECTIVE AND SUBJECTIVE

\begin{tabular}{|c|c|c|c|}
\hline \multicolumn{4}{|c|}{$\begin{array}{r}\text { PARAMETERS } \\
\end{array}$} \\
\hline $\begin{array}{c}\text { Objective } \\
\text { parameters }\end{array}$ & $\begin{array}{c}\text { GPRS } \\
\text { Percentage } \\
(G P)\end{array}$ & $\begin{array}{c}\text { Mod. Exercise } \\
\text { Percentage }(\mathrm{MEP})\end{array}$ & $\begin{array}{l}\text { Number of } \\
\text { Players }(P)\end{array}$ \\
\hline QoE & $\begin{array}{l}\mathrm{r}=-0.495 \\
\mathrm{p}=0.000\end{array}$ & $r=0.718 p=0.000$ & $\begin{array}{l}r=0.540 \\
p=0.000\end{array}$ \\
\hline Boredom (B) & $\begin{array}{l}r=0.377 \\
p=0.004\end{array}$ & $r=-0.672 p=0.000$ & $\begin{array}{l}r=-0.501 \\
p=0.000\end{array}$ \\
\hline Absorption (A) & & $r=0.676 p=0.000$ & \\
\hline
\end{tabular}

\section{3) QoE model}

The regression model of (1) expresses the overall quality of experience (QoE) of a location-based mobile MMORPG on a 5-point scale based on the amount (in percentage) of moderate exercise (instead of light vigorous or very vigorous) performed by the user while playing PK $(M E P)$, the percentage of the gaming session the user used a GPRS connection $(G P)$ and the number of players around the test user in the virtual world $(P)$. Based on the correlations listed in Table I, the following model is presented:

$$
\mathrm{QoE}=1.629+0.019 \cdot \mathrm{MEP}-0.045 \cdot \mathrm{GP}+0.300 \cdot \mathrm{P}
$$

QoE is a discrete number [1..5]. Both $M E P$ and $G P$ in (1) are expressed as a percentage value, whereas $P$ is a number expressing the number of players in the virtual world. This model of QoE presents 0.67 as coefficient of determination (R2). R2 stands for the proportion of variability in the data set that is accounted for by the statistical model.

The model evidences the influence of the interaction with other players in a mobile location-based MMORPG. The number of test users the player can chat, sell or buy items, plan strategies, etc. with, has a significant impact in the overall QoE. A higher $G P$ implies a worse quality network connection, because the users were playing PK connected to a GPRS connection instead of to a faster data connection (e.g. UMTS). Therefore, GP negatively influences the overall QoE, even if the users did not consciously realize any difference. Finally, the QoE is positively affected by the amount of moderate exercise performed by the user, MEP in (1). The dependence on MEP of the overall QoE related to $\mathrm{PK}$ is perfectly understandable because of the fact that users are able to access new virtual areas as they move in the real world. Hence, assuming that the physical activity that the players underwent was in order to move out of their virtual circle of mobility, physical activity implies a higher chance of exploring new virtual areas in which the user will be able to find new resources, monsters to hunt and territories to conquer.

\section{4) Boredom model}

The following model is presented, based on the correlations listed on Table I:

$$
\mathrm{B}=4.016+0.028 \cdot G P-0.024 \cdot \mathrm{MEP}-0.258 \cdot \mathrm{P}
$$

$\mathrm{B}$ is a discrete number [1..5]. Both $M E P$ and $G P$ in (2) are expressed as a percentage value, whereas $P$ is a number expressing the number of players in the virtual world. Boredom (B) experienced by a user while playing a location-based 
mobile MMORPG on a 5-point scale is estimated by the regression model of (2) based on the amount (in percentage) of moderate exercise performed by the user while playing PK $(M E P)$ and the number of players around the test user in the virtual world $(P)$. The coefficient of determination (R2) of this model equals 0.54 .

Conclusions drawn from this regression model are very similar to those explained in the previous section since apparently this feeling of boredom and how enriching the user found the gaming experience $(\mathrm{QoE})$ are opposing parameters. These two parameters present a highly significant $(\mathrm{p}=0.000)$ negative correlation $(\mathrm{r}=-0.608)$ and their regression models are very similar.

\section{5) Absorption model}

Finally, the following model is inferred taking into account the correlations found in section E.2:

$$
\mathrm{A}=1.362+0.039 \cdot \mathrm{MEP}
$$

$\mathrm{A}$ is a discrete number [1..5]. $M E P$ is expressed as a percentage value. The regression model of (3) expresses the absorption $(A)$ experienced by a user while playing a locationbased mobile MMORPG on a 5-point scale based on the percentage of moderate exercise performed by the user while playing PK (MEP). The current model has an R2 value of 0.45 . The absorption felt by a user is significantly affected by the amount of moderate exercise performed by the user $(M E P)$. This parameter was not influenced by $G P$ or $P$ as these two parameters did not significantly correlate with the Absorption (A).

\section{6) Evaluation of curve fitting}

In order to evaluate the curve fitting of the proposed models, a prediction of the QoE, Boredom and Absorption was calculated with (1), (2), and (3), respectively, for each gaming session of the experiment. Subsequently, these predictions were compared with the actual QoE value, boredom level and absorption felt while playing PK expressed by the end-users through the questionnaires.

\begin{tabular}{cccc}
\multicolumn{2}{c}{ TABLE II. } & Evaluation OF THE REGRESSION MODELS \\
\hline & QoE Model & Boredom Model & $\begin{array}{c}\text { Absorption } \\
\text { Model }\end{array}$ \\
\hline $\begin{array}{c}\text { Number Of } \\
\text { Predictions }\end{array}$ & 56 & 56 & 56 \\
\hline RMSE & 0.71 & 0.87 & 0.87 \\
\hline Correct predictions & $31[55.3 \%]$ & $22[39.3 \%]$ & $23[41.1 \%]$ \\
\hline 1 star deviation & $24[42.8 \%]$ & $32[57.1 \%]$ & $30[53.6 \%]$ \\
\hline 2 stars deviation & $1[1.9 \%]$ & $2[3.6 \%]$ & $3[5.3 \%]$ \\
\hline
\end{tabular}

Table II summarizes the results of this evaluation by mentioning the number of predictions based on each model together with the root mean square error (RMSE) of these predictions with respect to the actual values of the aforementioned subjective values. Table II lists the number of rounded predictions which equal the actual values (reported as correct predictions). The incorrect predictions are classified according to their deviation from the actual value $(1,2,3$, or 4 stars deviation). Table II indicates that none of the predictions ( 3 for the content model and 6 for the interest model) suffer from a deviation of more than 2 stars, which proves the accuracy of the models.

\section{CONCLUSION}

In this paper a fully interdisciplinary QoE model is created taking into account wireless infrastructure and network QoS indicators, player's context and physiological data obtained from on-body sensors as QoE factors. Both, a methodology and a model to estimate QoE of mobile location based MMORPGs are presented based on the statistical analysis of this particular mobile game. The concept and implementation of a re-usable QoE measurement framework on Android platform is discussed. The result of the measurements is analyzed and the influence of the different QoE factors on the overall QoE and subjective feelings of absorption and amusement related to mobile gaming is investigated. The results show that both the physical activity a player undergo and the interaction with other players affects positively the QoE of a location-based MMORPG whereas a worse quality network connection has a negative impact on the QoE. In addition, these parameters affect the same way the feeling of boredom experienced by a user while playing a location-based MMORPG. Finally, we conclude that how absorbed the user felt in the mobile game is influenced by the physical activity a player underwent.

\section{REFERENCES}

[1] D. Lopez, et al., Adaptive multimedia streaming over IP based on customer oriented metrics, ISCN, pp. 185-191 2006.

[2] K. De Moor et al., Proposed Framework for Evaluating Quality of Experience in a Mobile, Testbed-oriented Living Lab Setting, Mobile Networks and Applications, 2010.

[3] K. Kilkki, Quality of Experience in Communications Ecosystem, Journal of Universal Computer Science, vol. 14, p. 615, 2008.

[4] Moon-Hee Park et al., Location-Based Recommendation System Using Bayesian User's Preference Model in Mobile Devices, Lecture Notes in Computer Sciences, vol. 4611, pp. 1130-1139, 2007.

[5] Brown, B. et al., Sharing the Square: Collaborative Leisure in the City Streets, Proc. ECSCW, pp. 427-447, 2005.

[6] Flintham, M. et al., Where on-line meets on-thestreets: experiences with mobile mixed reality games, Proceedings of the SIGCHI conference on Human factors in computing systems, pp. 569-576, 2003.

[7] Marek Bell et al., Interweaving Mobile Games With Everyday Life, Proceedings Games and Performances, pp.417-426, 2006

[8] Adriana de Souza e Silva, Alien Revolt (2005-2007): A Case Study of the First Location-Based Mobile Game in Brazil, IEEE Technology and Society Magazine, vol. Spring 2008, pp. 18-28, 2008

[9] C. Licoppe, and Y. Inada, Emergent uses of a multiplayer locationaware mobile game: The interactional consequences of mediated encounters, Mobilities, vol. 1, pp. 39-61, Mar. 2006.

[10] Yu-Chun Chang, Kuan-Ta Chen, Chen-Chi Wu, Chien-Ju Ho, and ChinLaung Lei, Online Game QoE Evaluation using Paired Comparisons, In Proceedings of IEEE CQR 2010, June 2010.

[11] Vicente, K. J et al., Spectral Analysis of Sinus Arrhythmia: A Measure of Mental Effort, Human Factors, vol. 29, pp. 171 - 182, 1987.

[12] Ekman, P. et al., Autonomic Nervous System Activity Distinguishes among Emotions, Science, vol. 221, pp. 1208 -1210, 1983.

[13] Regan L.Mandryk et al., Using psychophysiological techniques to measure user experience with entertainment technologies, Journal of Behaviour and IT, vol. 25, pp. 141-158, 2005

[14] Parallel Kingdom, http://www.parallelkingdom.com/, 2010

[15] SenseWear BMS, http://www.sensewear.com/, 2010

[16] V. Jacobson, Tcpdump packet analyzer, The Tcpdump Manual Page, Lawrence Berkeley Laboratory, 1989

[17] Freedson, P. S., Melanson, E. L., Sirard, J. R., Calibration of the Computer Science and Applications, Inc. accelerometer, Journal of Med Sci Sports Exerc, vol 30, p. 777, 1998

[18] M. H. Kutner, C. J. Nachtsheim, J. Neter, and W. Li, Applied Linear Statistical Models, McGraw-Hill/Irwin, fifth edition, 2005 